\title{
Clinical Pharmacokinetics, Safety, and Tolerability of a Novel, First-in-Class TRPV4 Ion Channel Inhibitor, GSK2798745, in Healthy and Heart Failure Subjects
}

\author{
Navin Goyal ${ }^{1}$ (C) Pete Skrdla ${ }^{1} \cdot$ Rosemary Schroyer ${ }^{1} \cdot$ Subramanya Kumar $^{2} \cdot$ Disala Fernando $^{2} \cdot$ Anna Oughton $^{1}$. \\ Nicola Norton ${ }^{2} \cdot$ Dennis L. Sprecher ${ }^{1} \cdot$ Joseph Cheriyan ${ }^{2,3}$
}

Published online: 14 January 2019

(c) The Author(s) 2019

\begin{abstract}
Introduction and Objective Pulmonary capillary endothelial transient receptor potential vanilloid 4 (TRPV4) channel plays a critical role in mediating the development of cardiogenic pulmonary edema. GSK2798745 is a first-in-class, highly potent, selective, orally active TRPV4 channel blocker being evaluated in a first-time-in-humans study (NCT02119260).

Methods GSK2798745 was administered in a randomized, placebo-controlled study design to healthy volunteers in three separate cohorts as single escalating doses, with and without food, and as once-daily repeat doses for up to 14 days, respectively. Two cohorts of subjects with mild to moderate heart failure were also administered GSK2798745 once daily for up to 7 days. Safety, tolerability, and systemic exposure data were collected.

Results No significant safety issues or serious adverse events were observed with GSK2798745 in healthy volunteers and patients with heart failure. GSK2798745 systemic exposure data demonstrated linear pharmacokinetics up to $12.5 \mathrm{mg}$, less than twofold accumulation with once-daily dosing, and a systemic half-life of $\sim 13 \mathrm{~h}$. There was a slight increase in GSK2798745 exposure [14\% increase in area under the plasma concentration-time curve (AUC) and $9 \%$ increase in maximum observed plasma concentration $\left.\left(C_{\max }\right)\right]$ after administration with a high-fat meal.

Conclusions GSK2798745 was well-tolerated in healthy volunteers and patients with stable heart failure. The safety and exposure data obtained in this study allow further evaluation of the drug in long-term clinical studies in heart failure as well as other indications.
\end{abstract}

\section{Introduction}

Transient receptor potential vanilloid 4 (TRPV4) is an ion channel expressed in the pulmonary vasculature distributed through the alveolar septum. In isolated lung models, TRPV4 blockade prevented high pulmonary venous

Electronic supplementary material The online version of this article (https://doi.org/10.1007/s40256-018-00320-6) contains supplementary material, which is available to authorized users.

Navin Goyal

navinkumar.s.goyal@gsk.com

1 GlaxoSmithKline, UP4300 East, 1250 S College Road, Collegeville, PA 19426, USA

2 GlaxoSmithKline Clinical Unit Cambridge, Addenbrooke's Hospital, Cambridge, UK

3 Cambridge University Hospitals NHS Foundation Trust, Cambridge, UK pressure (PVP)-mediated increases in vascular permeability and lung edema [1]. Similarly, in an experimental model of myocardial infarction, use of a TRPV4 blocker prevented and reversed pre-established lung edema [1]. TRPV4 may also be involved in the regulation of vascular tone [2]. This approach, therefore, may have therapeutic values in congestive heart failure in humans.

GSK2798745 is a first-in-class, highly potent, selective, orally active TRPV4 channel blocker. The potency of GSK2798745, as assessed by measuring calcium influx induced by an in-house TRPV4 agonist using a fluorometric imaging plate reader (FLIPR)-based assay and recombinant human TRPV4 channels transduced into human embryonic kidney (HEK) cells, was in the range of $\sim 2-4 \mathrm{nM}$.

This was a first-time-in-humans (FTIH) phase I clinical trial for GSK2798745 that primarily evaluated the safety, tolerability, and pharmacokinetics (PK) of single and repeat oral doses of GSK2798745 administered to healthy subjects and patients with stable heart failure [New York 


\section{Key Points}

Transient receptor potential vanilloid 4 (TRPV4) is an ion channel expressed in the pulmonary vasculature distributed through the alveolar septum

In preclinical studies, a TRPV4 blocker administered before or after the induction of high pulmonary venous pressure resulted in the amelioration of the anticipated increase in lung edema, accompanied by an improvement in partial pressure oxygen $\left(\mathrm{PO}_{2}\right)$

GSK2798745 is a first-in-class, highly potent, selective, orally active TRPV4 channel blocker

GSK2798745 was well tolerated in healthy volunteers and subjects with mild-moderate heart failure during single and once-daily repeat dosing

GSK2798745 can be administered as a once-daily regimen without any meal restrictions

Relatively high levels of TRPV4 channel blockade activity can be achieved at the dosing regimen studied

Heart Association (NYHA) class II-III]. The results from these primary endpoints are presented in this manuscript. The clinical data generated in the current study lay the foundation for further exploration of GSK2798745 in various dosing regimens and other indications in future clinical studies.

\section{Methods}

\subsection{Study Design, Treatment, and Patient Eligibility}

This was a randomized, double-blind (sponsor unblinded) study with five cohorts that included healthy volunteers and subjects with stable heart failure as outlined in Fig. 1. The safety and PK of GSK2798745 after single-dose escalation, relative bioavailability of liquid and capsule formulations with and without food, and up to 14-day repeat doses in healthy subjects were investigated in cohorts 1, 2, and 3, respectively. The safety and PK of GSK2798745 in patients with stable heart failure were evaluated in cohort 4 (single and repeat dose) and cohort 5 (repeat dose only). Cohorts 4 and 5 were an early safety study, which included patients with mild to moderate heart failure of any etiology with symptoms corresponding to NYHA class II or III on stable heart failure therapy for at least 1 month without recent hospitalization for heart failure in the preceding 3 months. This study was conducted primarily at one site, apart from cohort 4 which was conducted at two sites in the UK. The trial was granted favorable ethical opinion by the Oxford A REC ethics committee (14/SC/0220, Bristol, UK) and regulatory approval from the Medicines Healthcare Regulatory Agency for both clinical sites. Both studies complied with the Declaration of Helsinki 2008 and International Council for Harmonization good clinical practice guidelines, and full written informed consent was obtained from all participants before the performance of any study-specific procedures. A complete list of inclusion and exclusion criteria is available on clinicaltrials.gov (NCT02119260).

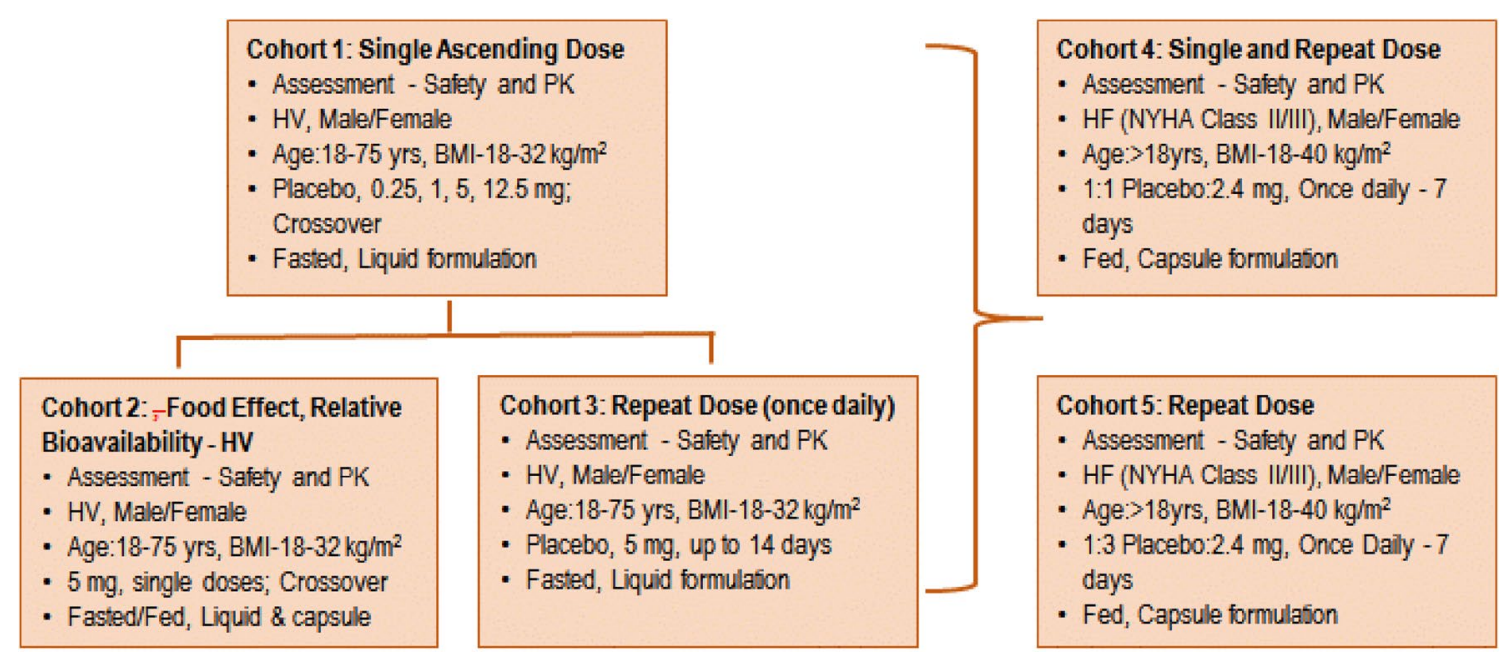

Fig. 1 Study schematic. Clinical study NCT02119260 study schematic. BMI body mass index, $H F$ subjects with stable heart failure, $H V$ healthy volunteers, NYHA New York Heart Association classification, $P K$ pharmacokinetics 
Dose escalation in cohort 1 was carried out with ongoing review of safety and PK data from lower doses. Each subject in this cohort participated in four treatment periods receiving three dosing sessions and placebo in one session with adequate washout of 7-21 days between treatment periods. Cohort 2 evaluated the relative bioavailability and food effects by employing a fixed sequence of liquid formulation (fasted), capsule (fasted), and capsule (fed) per US FDA food effect guidelines [3]. Cohort 3 enrolled healthy subjects to evaluate the PK of GSK2798745 at $5 \mathrm{mg}$ repeat doses administered as a liquid formulation for up to 14 days. Subjects were randomized at a ratio of $3: 1$ to receive active treatment or placebo. No drug was administered on Day 2 to allow for PK invariance assessments. Subjects were enrolled in cohorts 2 and 3 in parallel. During the progression of once-daily dosing in cohort 3 , the maximum individual exposure threshold targeted before study start was lowered based on emerging long-term preclinical safety data.

Ongoing analysis of preliminary safety and exposure data emerging from the healthy volunteers in cohorts 1-3 were employed to select the appropriate dose(s) and formulation to be administered in subjects with mild-moderate heart failure enrolled in cohorts 4 and 5. The subjects in cohort 4 were to receive a single dose of GSK2798745 or placebo (1:1 randomization ratio) followed by an adequate washout period of 1-3 weeks and to return for once-daily 7-day dosing with drug or placebo. A key exclusion criterion in this cohort was any contraindication to magnetic resonance imaging (MRI) scanning (e.g., pacemaker or intracranial or intraorbital metallic objects) since this was one of the pharmacodynamic measures in cohort 4. Cohort 5 included patients with mild-moderate heart failure randomized to receive active treatment or placebo in a 3:1 ratio to further evaluate the safety and PK of GSK2798745 after 7 days of once-daily repeat dosing. The exclusions relating to MRI applied in cohort 4 were removed for cohort 5 (e.g., patients with implanted pacemakers) as this group did not undergo MRI testing.

The initial dose escalation in cohort 1 was conducted by administering GSK2798745 as a liquid formulation. This was primarily in the form of drug powder in a bottle. Briefly, the dose strength determined was weighed in a bottle to which water was added and administered to the subject. The capsule formulation in cohorts 2,4 , and 5 were hand-filled capsules. The formulations were prepared at study site by the site pharmacist.

\subsection{Pharmacokinetic Assessments}

Blood samples were collected for PK analysis of GSK2798745 in all subjects. Samples were also collected to characterize circulating metabolites in all subjects, except for cohort 4 where metabolite identification samples were not collected. In cohorts 1 and 2, serial PK samples were collected from pre-dose to $72 \mathrm{~h}$ post-dose. In cohort 3, serial samples were collected on days 1 and 14, with some pre-dose samples on days 7 and 10. Similarly, serial PK samples were collected to adequately characterize the PK of GSK2798745 in subjects with heart failure in cohorts 4 and 5.

Plasma samples were analyzed for GSK2798745 by using a validated analytical method based on solid-phase extraction followed by high-performance liquid chromatography/tandem mass spectrometric detection (HPLC/MS/ MS) analysis. The lower limit of quantification (LLQ) was $0.400 \mathrm{ng} / \mathrm{mL}$ using a $200 \mu \mathrm{L}$ aliquot of $\mathrm{K}_{3}$ EDTA plasma. The higher limit of quantification (HLQ) was $100 \mathrm{ng} / \mathrm{mL}$. GSK2798745 bioanalysis was performed at GSK, UK, for the FTIH study. Each batch of experimental samples was run against duplicate freshly prepared calibration standards $(n=8)$. Calibration data were deemed acceptable if the backcalculated concentration did not deviate from the actual by more than $15 \%$ (20\% at LLQ) and if no more than $25 \%$ of the calibration standards were rejected or lost for any other reason. Quality control (QC) samples at three concentrations (two replicates per concentration) were also included in each run. Individual QC results were deemed acceptable if the calculated concentration deviated by no more than $15 \%$ from the actual concentration. The assay results were within acceptable limits with precision of 6.2-9.0\% across the QC concentration curve.

\subsection{Safety Assessments}

Safety and tolerability were assessed by monitoring of adverse events (AEs), clinical laboratory tests, and vital signs, including weight, resting pulse oximetry, electrocardiograms (ECGs, including telemetry monitoring), physical examinations, audiometry (cohorts 3-5), echocardiograms (at baseline and post dose, overread by a consultant echocardiologist), and remote cardiac monitoring using the BodyGuardian (Preventice Services, TX, USA) system (cohort 5). All AEs/serious AEs were collected from the time of administration of GSK2798745 until follow-up.

Based on preclinical data of GSK2798745, the drug was considered to possibly be central nervous system (CNS) active. There has been some concern that $\mathrm{CNS}$-active drugs may be associated with an increased risk of suicidal thinking or behavior in some subjects with certain conditions. Therefore, the Columbia Suicide Severity Rating Scale, a measure of suicidal ideation and behavior was included as a safety assessment in this study in subjects participating in the repeat-dose cohorts (cohorts 3-5). 


\subsection{Statistical Analysis}

The sample size for this FTIH study was primarily based on feasibility without formal power calculations. No formal statistical analysis of the safety data was performed. Plasma concentration-time data were analyzed by noncompartmental methods with Phoenix 6.0. From the plasma concentration-time data, the following PK parameters were determined: maximum observed plasma concentration $\left(C_{\max }\right)$, time to $C_{\max }\left(T_{\max }\right)$, area under the plasma concentration-time curve (AUC) from time zero to last quantifiable concentration $\left(\mathrm{AUC}_{(0-t)}\right)$ and from time zero to infinity $\left(\mathrm{AUC}_{(0-\infty)}\right)$, and apparent terminal phase half-life $\left(t_{1 / 2}\right)$. Calculations were based on the actual sampling times.

\section{Results}

\subsection{Healthy Volunteers}

The baseline demographics of healthy volunteer subjects randomized across cohorts 1-3 were similar and are listed in Table 1 in the Electronic Supplementary Material (ESM). All subjects were male with a mean age of approximately 42 years and a body mass index (BMI) of approximately $26 \mathrm{~kg} / \mathrm{m}^{2}$. Table 1 in the ESM also lists the number of subjects who were randomized in the study, completed, or withdrew from the study.
Cohort 1 planned to enroll eight subjects who received single GSK2798745 doses of 0.5, 1, 5, $12.5 \mathrm{mg}$ and/or placebo (six active, two placebo) with adequate washout in a crossover design. One subject in cohort 1 was withdrawn from the second treatment period before dose administration because of an AE (vasovagal syncope after intravenous cannulation). This subject was replaced with a new subject for the remaining treatment periods in cohort 1 . Thus, nine subjects were randomized in cohort 1 . The emerging safety and PK data from cohort 1 were evaluated before progressing to cohorts 2 and 3 in healthy volunteers.

A 5-mg dose was evaluated in 12 subjects in cohort 2 to assess the relative bioavailability and effect of food on GSK279845 exposure. One subject in the fasted state in this cohort 2 (treated with GSK2798745 suspension) withdrew consent for personal reasons after completing the first treatment period. As per protocol, this subject was not replaced, leaving 11 subjects completing the remaining two treatment periods.

Cohort 3, which was conducted in parallel to cohort 2, also evaluated the 5-mg dose of GSK2798745 to characterize its PK with a once-daily repeat-dosing regimen. Eight subjects received $5 \mathrm{mg}$ of GSK2798745 or placebo once daily (3:1 randomization ratio - six subjects on GSK2798745 and two subjects on placebo) for 14 days in this cohort 3 . Four subjects (three active, one placebo) completed the 14-day period, but the second set of four subjects (three active, one placebo) were dosed for 8 days when dosing in that cohort

Table 1 Pharmacokinetic parameters for GSK2798745 in healthy volunteer subjects

\begin{tabular}{|c|c|c|c|c|c|c|}
\hline Treatment & Subjects $(N)$ & Formulation & $C_{\max }{ }^{\mathrm{a}}(\mathrm{ng} / \mathrm{mL})$ & $\operatorname{AUC}_{(0-\infty)}{ }^{\mathrm{a}}(\mathrm{ng} \mathrm{h} / \mathrm{mL})$ & $T_{\max }{ }^{\mathrm{b}}(\mathrm{h})$ & $t_{1 / 2}^{\mathrm{a}}(\mathrm{h})$ \\
\hline \multicolumn{7}{|c|}{ Cohort 1 (single dose escalation) (fasted) } \\
\hline $0.25 \mathrm{mg}$ & 6 & Liquid & $1.1 \pm 0.4$ & $9.6 \pm 3.7$ & $1.0-2.0$ & $5.8 \pm 1.9$ \\
\hline $1 \mathrm{mg}$ & 6 & Liquid & $4.2 \pm 1.9$ & $62.2 \pm 26.6$ & $1.0-3.0$ & $13.4 \pm 4.4$ \\
\hline $5 \mathrm{mg}$ & 6 & Liquid & $22.9 \pm 2.4$ & $347.0 \pm 122.8$ & $2.0-3.0$ & $13.7 \pm 5.1$ \\
\hline $12.5 \mathrm{mg}^{\mathrm{c}}$ & 6 & Liquid & $47.9 \pm 12.1$ & $686.1 \pm 275.0$ & $2.0-3.0$ & $13.2 \pm 3.9$ \\
\hline \multicolumn{7}{|c|}{ Cohort 2 (food effect and relative bioavailability) } \\
\hline Fasted $5 \mathrm{mg}$ & 12 & Liquid & $22.2 \pm 5.7$ & $287.7 \pm 101.2$ & $1.0-3.0$ & $12.7 \pm 2.9$ \\
\hline Fasted $5 \mathrm{mg}$ & 11 & Capsule & $23.1 \pm 4.3$ & $312.1 \pm 71.0$ & $1.0-4.0$ & $12.9 \pm 2.7$ \\
\hline Fed $5 \mathrm{mg}$ & 11 & Capsule & $25.7 \pm 7.9$ & $361.8 \pm 99.4$ & $1.0-3.0$ & $13.8 \pm 2.5$ \\
\hline \multicolumn{7}{|c|}{ Cohort 3 (repeat dosing-once daily) } \\
\hline 5 mg (day 1 ) & 6 & Liquid & $23.9 \pm 5.6$ & $223.2 \pm 31.0^{\mathrm{d}}$ & $2.0-3.0$ & NA \\
\hline $5 \mathrm{mg}$ (day 14) & 3 & Liquid & $29.9 \pm 3.9$ & $318.7 \pm 39.6^{\mathrm{d}}$ & $2.0-2.0$ & NA \\
\hline
\end{tabular}

$A U C$ area under the plasma concentration-time curve, $C_{\max }$ maximum observed plasma concentration, NA not applicable, NOAEL no-observedadverse-effect level, $P I B$ powder in a bottle administered as solution/suspension, $T_{\max }$ time to $C_{\max }, t_{1 / 2}$ terminal-phase half-life

${ }^{a}$ All values are mean \pm standard deviation

${ }^{\mathrm{b}}$ Range of $T_{\max }$

${ }^{c}$ Exposure with the single dose of $12.5 \mathrm{mg}$ exceeded the limitations; this dose group was conducted with an earlier safety margin based on a NOAEL established in a 1-month dog toxicity study. The updated safety margin was based on the NOAEL established in a 3-month dog toxicity study

${ }^{\mathrm{d}}$ AUC over 24-h time period only 
was terminated based on the emerging data from a concurrent long-term preclinical safety study. It should be noted that, although a placebo subject in cohort 3 is included in Table 6 as having withdrawn consent, this was after completion of 8 days of dosing as the subject did not wish to complete the 72-h follow-up period.

\subsection{Subjects with Heart Failure}

Cohort 4 enrolled a total of 23 subjects with mild-moderate heart failure with ten subjects randomized to GSK2798745 and 13 subjects to placebo. Cohort 5 enrolled a total of eight subjects, six randomized to GSK2798745 and two randomized to matching placebo. All subjects in these cohorts completed the study as summarized in Table 2 in the ESM.

The baseline demographics of the subjects with heart failure were generally similar between the group receiving 2.4-mg repeat doses of GSK2798745 and the placebo group in cohorts 4 and 5 (Table 2 in the ESM). The subjects in both cohorts had a mean age of approximately 67 years and a BMI of approximately $29 \mathrm{~kg} / \mathrm{m}^{2}$ (cohort 4) and $27 \mathrm{~kg} / \mathrm{m}^{2}$ (cohort 5). All subjects in cohort 5 were male, and 7 of the 23 subjects in cohort 4 were female.

\subsection{Pharmacokinetics in Healthy Subjects}

The average concentration-time profiles for ascending GSK2798745 single doses are presented in Fig. 2. The summary PK parameters in healthy subjects in cohorts 1-3 are listed in Table 1. Dose escalation in cohort 1 demonstrated approximate dose-proportional PK for doses from 1 to $12.5 \mathrm{mg}$ for $C_{\max }$ and AUC. GSK2798745 was rapidly absorbed with $T_{\max }$ between 1 and $4 \mathrm{~h}$, with an approximate $t_{1 / 2}$ of $13 \mathrm{~h}$. TRPV4 channel blockade can be estimated corresponding to any systemic concentration and the potency $(\sim 2-4 \mathrm{nM})$ derived from preclinical experiments. The observed GSK2798745 peak systemic exposures at the single doses $\geq 5 \mathrm{mg}$ were estimated to provide $>90 \%$ TRPV4 channel blockade based on its high potency. A once-daily dosing regimen in cohort 3 led to less than twofold drug accumulation as seen from the exposure data. Based on

Table 2 Impact of formulation and food on the systemic exposure of GSK2798745 $5 \mathrm{mg}$

\begin{tabular}{lll}
\hline Comparison & $\mathrm{AUC}_{(0-\infty)}$ ratio & $C_{\max }$ ratio \\
\hline Fasted capsule to fasted PIB & $1.12(0.96-1.29)$ & $1.05(0.92-1.20)$ \\
Fed capsule vs. fasted capsule & $1.14(1.02-1.28)$ & $1.09(0.93-1.28)$ \\
\hline
\end{tabular}

Data are presented as mean ( $90 \%$ confidence interval)

$A U C$ area under the plasma concentration-time curve, $C_{\max }$ maximum observed plasma concentration, PIB GSK2798745 administered as a powder in bottle (suspension) formulation trough concentrations, steady state appeared to be attained in approximately 4-6 days with the once-daily dosing regimen.

The systemic exposure data from subjects in cohort 2 demonstrated a small increase in the $C_{\max }(4 \%)$ and AUC (12\%) when a 5-mg dose of GSK2798745 was administered as a capsule compared with the liquid formulation. Further administration of 5-mg GSK2798745 capsules with a standard FDA-recommended high-fat meal resulted in an increase of $9 \%$ in $C_{\max }$ and $14 \%$ in AUC compared with administration in the fasted state. These changes are summarized in Table 2 and were not considered clinically relevant.

\subsection{Pharmacokinetics in Subjects with Heart Failure}

All available data from healthy subjects in the first 3 cohorts were used to develop a preliminary population PK model to simulate exposures in subjects with heart failure recruited in cohorts 4 and 5 . In addition, preliminary data obtained from cohort 2 indicated small increases $(\sim 25 \%)$ in systemic exposure when GSK2798745 was administered with food as a capsule formulation. Subsequently, a 2.4-mg capsule formulation with food was determined as the highest dose predicted to provide exposures with the lowest likelihood of any subject exceeding exposure thresholds established based on data from preclinical safety studies. Thus, a 2.4-mg dose in capsules without meal restrictions was recommended to be administered to subjects in cohorts 4 and 5 .

In cohort 4 , six patients initially received a single dose of a 2.4-mg GSK2798745 capsule or placebo in period 1 followed by a 1-week washout before starting period 2 to evaluate the safety, tolerability, and PK of once-daily 7-day repeat dosing of GSK2798745. Based on preliminary safety data in the first six subjects, subsequent new patients enrolled in cohort 4 were directly randomized to receive once-daily 7-day repeat doses of $2.4 \mathrm{mg}$ of GSK2798745 or placebo.

The PK results obtained from the patients with mildmoderate heart failure in these two cohorts are summarized in Table 3, and the day 1 profiles are presented in Fig. 2. The PK data in cohort 4 were obtained from few subjects and thus the PK parameters could not be adequately or reliably estimated in every subject. However, the limited data from period 1 indicated a systemic mean \pm standard deviation (SD) for half-life of approximately $17 \pm 4.5 \mathrm{~h}$, with a $T_{\max }$ ranging from 1 to $6 \mathrm{~h}$ (data on file). Systemic exposure of GSK2798745 in cohort 5 demonstrated less than twofold accumulation with the once-daily 7-day dosing regimen. The PK sampling scheme did not allow estimation of the $\mathrm{t}_{1 / 2}$ in this cohort. The $T_{\max }$ ranged from 1 to $5 \mathrm{~h}$ and was comparable to that observed in cohort 4 . Overall, the average exposures observed in subjects with heart failure were approximately $20 \%$ higher than those in healthy subjects. More data may be needed to reliably quantify such small differences. The peak exposures observed on days 1 and/ 
Fig. 2 GSK2798745 single dose concentration-time profiles in healthy volunteers and stable heart failure subjects; Mean+SD. Single dose data only; $H V$ healthy volunteers, $H F$ subjects with stable heart failure, $P 1 D 1$ period 1 day $1, P 2 D 1$ period 2 day $1, D 1$ day 1

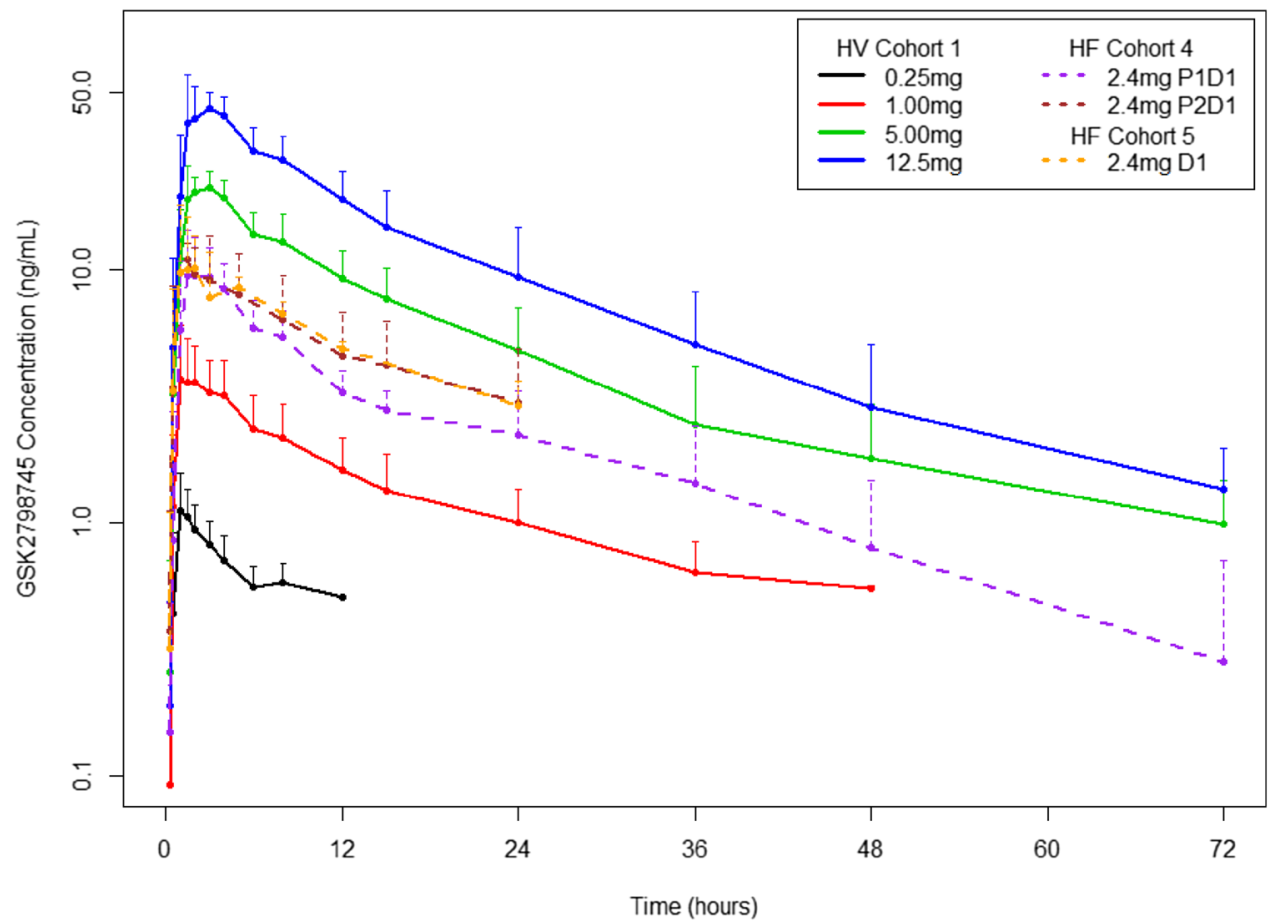

Table 3 Pharmacokinetic parameters from cohort 4 heart failure subjects (2.4-mg capsule once daily with food)

\begin{tabular}{lllc}
\hline Parameter & Day & $n$ & Mean $\pm \mathrm{SD}$ \\
\hline Cohort $4^{\mathrm{a}}$ & & & \\
$C_{\max }(\mathrm{ng} / \mathrm{mL})$ & 1 & 4 & $13.7 \pm 1.9$ \\
& 7 & 9 & $17.1 \pm 7.3$ \\
$\mathrm{AUC}_{(0-24)}(\mathrm{h} * \mathrm{ng} / \mathrm{mL})$ & 1 & 4 & $133.6 \pm 57.9$ \\
& 7 & 9 & $208.5 \pm 115.2$ \\
Cohort 5 & & & \\
$C_{\max }(\mathrm{ng} / \mathrm{mL})$ & 1 & 6 & $13.4 \pm 4.1$ \\
$\mathrm{AUC}_{(0-24)}(\mathrm{h} * \mathrm{ng} / \mathrm{mL})$ & 1 & 6 & $16.2 \pm 3.5$ \\
& 7 & 6 & $131.4 \pm 11.5$ \\
\hline
\end{tabular}

$A U C$ area under the plasma concentration-time curve, $C_{\max }$ maximum observed plasma concentration, $S D$ standard deviation

${ }^{\mathrm{a}}$ Data for cohort 4 are from period 2

or 7 in these cohorts were adequate to provide a predicted 80-90\% TRPV4 channel blockade on average.

\subsection{Safety Results}

Single and repeat doses of GSK2798745 between 0.25 and $12.5 \mathrm{mg}$ were generally well tolerated in healthy volunteer subjects. A majority of subjects reported single AEs in cohorts 1-3, and all AEs were mild to moderate in intensity. One subject in cohort 1 was withdrawn from the second treatment period before dose administration due to an $\mathrm{AE}$ of vasovagal syncope after intravenous cannulation. This subject received $0.25 \mathrm{mg}$ of GSK2798745 in the first treatment period. As per protocol, the withdrawn subject was replaced by a subject who received the remaining two doses of GSK2798745 in the sequence. Back pain and dyspnea were reported by two subjects each after treatment with the 5-mg liquid formulation of GSK2798745. No clinically significant safety concerns were observed with administration of GSK2798745 after single and repeat doses to healthy volunteers, and no serious AEs were reported in any of the subjects. AEs reported with single or repeat administration to healthy volunteers that were considered related to treatment with $5 \mathrm{mg}$ of GSK2798745 included headache (two subjects), nasopharyngitis, dizziness, fatigue, nasal congestion, dyspepsia, and feeling abnormal (each in one subject). The detailed AE listings for cohorts 1,2, and 3 are provided in Tables 3, 4, and 5 in the ESM.

Similarly, repeat doses of $2.4 \mathrm{mg}$ of GSK2798745 for up to 7 days were also generally well-tolerated in patients with heart failure (Table 6 in the ESM). No clinically significant safety concerns were observed with administration of GSK2798745 to patients with heart failure. Furthermore, none of these patients were withdrawn from the study because of AEs. Within cohort 4, one subject who received $2.4 \mathrm{mg}$ of GSK2798745 experienced an accelerated idioventricular rhythm that was considered drug related by the investigator. There were no reports of drug-related AEs in patients with heart failure in cohort 5. It should be noted that nonsustained ventricular tachycardia in these patients was frequently present at pre-dose. 
Based on ECGs, telemetry (continuous to $24 \mathrm{~h}$ after single-dose administration in cohorts 1,2 , and 4 and up to $48 \mathrm{~h}$ after repeat dosing in patients with heart failure in cohorts 4 and 5), pulse oximetry, vital sign assessments, clinical laboratory assessments (including troponin), echocardiograms, weight measurements, appetite assessments, and suicidality questionnaires, no clinically significant safety concerns were noted. An oral glucose tolerance test administered in cohort 3 (four subjects who completed the scheduled 14-day repeat period) showed no indication of hyperglycemia.

The majority of subjects with heart failure in each treatment group in cohorts 4 and 5 did not report a change from baseline in audiometry. The proportion of subjects with heart failure with a change from baseline in audiometry was low and similar between the treatment groups. Other exploratory assessments were conducted in the repeat-dose cohorts 3-5 in healthy volunteers and subjects with heart failure, which will be reported separately.

\section{Discussion}

The safety and PK of GSK2798745 was evaluated in this FTIF study in healthy volunteers and subjects with mild to moderate stable heart failure. TRPV4 is a mechanosensitive cation channel, which promotes dramatic shifts in intracellular calcium, altering intracellular signaling and endothelial cytoskeleton status when activated by hypotonicity, stretch, pressure, temperature shifts, or various ligands, such as phorbol esters and arachidonic acid metabolites, e.g., epoxyeicosatrienoic acids (EETs) [4]. While TRPV4 channel is found in the alveolar:vascular membrane, it is also wellrepresented in endothelial and epithelial cells throughout the vasculature, along the mucosal surface of the gastrointestinal tract, renal tubule, and the choroid plexus of the brain. Therefore, pressure and stretch can activate the channel and hypothetically produce changes in lung, renal, gastrointestinal, or potentially cerebrospinal fluid distribution, thereby leading to clinical manifestations. The manipulation of a systemically ubiquitous channel such as TRPV4, specifically given its role in calcium trafficking, could potentially result in deleterious clinical signs and symptoms. Activation of TRPV4 in response to increased PVP can cause endothelial cell contraction and detachment, reducing the integrity of the alveolar barrier, increasing permeability, leading to pulmonary edema and impairment at the alveolar:vascular interface, leading to dyspnea. While the exact mechanism of action of TRPV4 inhibitors is not known, the preclinical in vitro and in vivo studies with elective TRPV4 channel blockers support this mechanism of action [4-6].

The healthy volunteer cohorts demonstrated no detectable changes in vital signs, clinical laboratory tests, or physical examinations. The single and repeat administration of
GSK2798745 at doses ranging from 0.25 to $12.5 \mathrm{mg}$ were generally well-tolerated in healthy volunteer subjects. The starting doses for this study were selected based on all available preclinical data and in accordance with the regulatory guidance $[7,8]$. Emerging safety and PK data were used to guide dose escalation for subsequent arms and cohorts. No clinically significant safety concerns were identified related to GSK2798745 administration in this study with $12.5-\mathrm{mg}$ single doses and 5-mg once-daily repeat doses for up to 14 days. The exposures at these higher GSK2798745 doses (i.e., $\geq 5 \mathrm{mg}$ ) administered as a single dose or once daily for up to 14 days are predicted to provide near complete TRPV4 channel blockade with the $2-4 \mathrm{nM}$ potency.

The dose escalation in healthy volunteers demonstrated approximate dose proportionality in PK from 1 to $12.5 \mathrm{mg}$ doses. GSK2798745 was rapidly absorbed, with a $T_{\max }$ of between 1 and $4 \mathrm{~h}$ with a less than twofold accumulation with the once-daily repeat-dose regimen. The mean systemic half-life of GSK2798745 was $\sim 13 \mathrm{~h}$. The increase in systemic exposure (AUC 14\%; $C_{\max } 9 \%$ ) when administering GSK2798745 with a standard FDA-recommended high-fat meal was clinically insignificant. Thus, future studies can dose GSK2798745 without any meal restrictions.

The emerging data from healthy volunteers allowed administration of GSK2798745 with meals to subjects with stable heart failure in cohorts 4 and 5 . The slightly higher exposures in these cohorts may need to be qualified with additional systemic exposure data from other clinical studies. A population analysis of GSK2798745 is already underway by combining the drug's systemic exposure data from this study and a relative bioavailability study comparing three different formulations in healthy volunteers, and this will be reported separately. The population analysis approach potentially allows PK characterization by pooling exposure data across different dosing regimens, study populations, and formulations. There appears to be no clinically relevant PK differences across healthy volunteers and subjects with heart failure.

The disease pathophysiology in subjects with heart failure presented some uncertainty and risk in administering a TRPV4 channel blocker to these patients as compared with healthy volunteers. For example, while a TRPV4 channel blocker could impede the fluid movement, perhaps providing symptomatic benefit, it could also impede fluid transport back out into the vasculature from within the interstitial space. This might have led to increased shortness of breath or reduced exercise tolerance along with consequent pulmonary edema. While the study inclusion criteria allowed enrollment of subjects with NYHA class II and III, almost all subjects enrolled in this study were relatively stable, well-treated class II subjects. They showed no worsening of heart failure symptoms nor changes in physical examination. The subjects with heart failure, with presumed 
baseline abnormalities in interstitial fluid levels, also did not reveal physical, clinical, or laboratory abnormalities with the observed GSK2798745 exposures from the single or once-daily 7-day repeat-dose administration. The exposures observed at the administered doses are predicted to provide high levels of target engagement of $80-90 \%$ and 60-70\% TRPV4 channel blockade at peak and trough exposures, respectively.

\section{Conclusion}

In summary, the current study adequately characterized the safety and PK of GSK2798745 at the observed exposures with administered doses in healthy subjects and patients with stable heart failure. GSK2798745 was well-tolerated, without any clinically significant safety concerns at the various doses and regimens evaluated in the study. These data will allow for a selection of appropriate doses from the current dosing regimen for further longer-term clinical evaluation of GSK2798745 in patients with heart failure. Given the ubiquitous nature of the TRPV4 channel as a target, the data also allow clinical evaluation of GSK2798745 in different indications.

Acknowledgements The authors thank all the patients, volunteers, and staff at the GSK Clinical Unit Cambridge whose dedication and time contributed to the success of this study. We also thank Lea Sarov-Blat for all her contributions in the initial design of the study. Anonymized individual participant data and study documents can be requested for further research from http://www.clinicalstudydatarequest.com.

Author Contributions NG, AO, PS, RS, DLS, and JC conceived and designed the study. JC, SK, DLS, and DF were involved with the oversight and conduct of the clinical study. NN, SK, DF, and JC were involved in clinical study execution and data collection. NG, RS, DLS, and JC were involved with data analysis. All authors contributed to the manuscript.

\section{Compliance with Ethical Standards}

Funding Funding for this study TR4117387 (NCT02119260) was provided by GlaxoSmithKline. GlaxoSmithKline was involved in all aspects of this study, including design, conduct, data collection, data management, data analysis, data interpretation, and the preparation, review, and approval of the manuscript.

Conflict of Interest All authors meet the criteria for authorship set forth by the International Committee for Medical Journal Editors. NG,
PS, RS, SK, DF, and NN are employees of GSK and hold stocks in the company. The chief investigator for the study was JC, who is a Cambridge University Hospitals National Health Service Foundation Trust employee, obligated via a secondment from his employer to spend $50 \%$ of his time on clinical trials activities for GSK; however, he receives no employee benefits from GSK. JC acknowledges funding support from the National Institute for Health Research (NIHR) Cambridge Biomedical Research Centre, Cambridge University Hospitals. The views expressed are those of the authors and not necessarily those of the NHS, the NIHR or the Department of Health and Social Care. AO and DLS were GSK employees at the time of the study and held stocks in the company; they have since both left the company. Editorial support (development of the first draft, assembling tables, collating author comments, and referencing) was provided by Guissou Dabiri, $\mathrm{PhD}$, of GD Scientific \& Medical Writing, LLC, and was funded by GSK.

Open Access This article is distributed under the terms of the Creative Commons Attribution-NonCommercial 4.0 International License (http://creativecommons.org/licenses/by-nc/4.0/), which permits any noncommercial use, distribution, and reproduction in any medium, provided you give appropriate credit to the original author(s) and the source, provide a link to the Creative Commons license, and indicate if changes were made.

\section{References}

1. Thorneloe KS, Cheung M, Bao W, et al. An orally active TRPV4 channel blocker prevents and resolves pulmonary oedema induced by heart failure. Sci Transl Med. 2012;4(159):159ra148.

2. Yang L, Maki-Petaja K, Cheriyan J, McEniery C, Wilkinson IB. The role of epoxyeicosatrienoic acids in the cardiovascular system. Br J Clin Pharmacol. 2015;80:28-44.

3. US Food and Drug Administration Guidance for Industry. Foodeffect bioavailability and fed bioequivalence studies. December 2002.

4. Everaerts W, Nilius B, Owsianik G. The vallinoid transient receptor potential channel TRPV4: from structure to disease. Prog Biophys Mol Biol. 2010;103:2-17.

5. Alvarez DF, King JA, Weber D, Addison E, Liedtke W, Townsley MI. Transient receptor vanilloid 4-mediated disruption of the alveolar septal barrier: a novel mechanism of acute lung injury. Circ Res. 2006;99:988-95.

6. Jian MY, King JA, Al-Mehdi AB, Liedtke W, Townsley MI. High vascular pressure-induced lung injury requires $\mathrm{P} 450$ epoxygenasedependent activation of TRPV4. Am J Respir Cell Mol Biol. 2008;38(4):386-92.

7. Committee for Medicinal Products for Human Use (CHMP). Guideline on Strategies to Identify and Mitigate Risks for FirstInhuman Clinical Trials with Investigational Medicinal Products. 1 September 2007.

8. US Food and Drug Administration Guidance for Industry. Estimating the Maximum Safe Starting Dose in Initial Clinical Trials for Therapeutics in Adult Healthy Volunteers. July 2005. 